\title{
Does Citizen Science Biological Recording Tell Us As Much About The Recorders As Biodiversity?
}

\author{
Paul J. Palmer, Michael Henshaw, Russell Lock, \\ Alan Cann, David Nicholls
}

5th May 2020

\begin{abstract}
1. There is growing body of evidence that a connection to nature is important at both an individual emotional level, and for the development of societal attitudes that rank conservation issues as important, however, there is widespread loss of this connection in modern lifestyles. The recruitment of community stakeholders into 'Citizen Science' biodiversity projects is one mechanism that may help to restore that connection, while at the same time providing outcomes of scientific value. However, the narrative describing such projects tends to use a fundamentally Apollonian view of science, which casts the researcher as an impartial observer and operates within a carefully designed methodology to guide the contributions from volunteer stakeholders. While this is a convenient model for formally reporting scientific outcomes, it masks individual immersive engagement.

2. The opposing Dionysian viewpoint has proved capable of making contributions to many domains of human activity, but does it work with the citizen science paradigm? We explore this question by reviewing an established project, NatureSpot, that labels itself Citizen Science, and follows Dionysian principles where the contributors of natural history observations are self-motivated and self-appointed. Given the lack of formal structure it would be easy to write off the data collated as without scientific value, but we show that it is possible to infer a quasi-methodology for subsets of data by deducing quantifiable observer behaviours and incorporating them into the interpretation.

3. The conclusions acknowledge that the lack of a formal data collation methodology along with the existence of biases due to unknown causes are problematic. However, the large size of the data allows for statistical explorations that otherwise would not be practicable. The findings of this work show that proxies for observer expertise and geospatial species distribution can be derived from the data and allow insights into recorder behaviour that add value to the overall interpretation. Our findings suggest that good citizen science projects may be designed around Dionysian principles by appealing to individual stakeholder motivations, complementing the structured methodology preferred by the Apollonian approach.
\end{abstract}

Keywords: Citizen Science; Biodiversity Recording; Data Quality. 


\section{Introduction}

There is growing body of evidence that a connection to nature is important at both an individual emotional level, and for the development societal attitudes that place importance on the natural world. The negative impact of declining interaction with nature are examined in detail by Soga and Gaston (2016), who collate evidence from multiple sources and countries showing that this problem is widespread in modern lifestyles. This is not a new phenomenon, in his memoir Pyle (1993) refers to this as an 'extinction of experience', a term that eloquently evokes the sense of loss involved. These interactions may also be grouped within 'Cultural Ecosystem Services' (CES), a term which seeks to include the intangible benefits of ecosystems, defined by Sarukhán and Whyte (2005) as:

These are the non-material benefits people obtain from ecosystems through spiritual enrichment, cognitive development, reflection, recreation, and aesthetic experiences... .

While the intangible nature makes review difficult, Milcu et al. (2013) showed that such benefits may be measured in more than just monetary values. It is possible that the recruitment of community stakeholders into 'Citizen Science' biodiversity projects is one mechanism that may help to restore the beneficial connection to nature, while at the same time providing outcomes of scientific value.

Support for this idea is provided by Fish et al. (2016) who used a mixed methodology study to illustrate the many benefits of engagement. A particular observation relates to the plurality of methodology needed to uncover the full range of benefits from CES. These include emotional, social and societal on top of scientific benefits, which leads us to the conclusion that they must be included when considering citizen science projects. There is a tendency to regard 'good science' as always following a dispassionate structured methodology, but this is not necessarily the case. For example, Scheffer et al. (2015) argue the case for all scientists to include creative thinking processes in their work. They suggest that creative thinking taps into different psychological aspects of individuals and that scientific research benefits from a broader approach. These ideas were nicely encapsulated in a letter to Science by Szent-Györgyi (1972). He suggested that an Apollonian view of science tends to develop established lines of research to perfection, while the Dionysian view relies on intuition and is more likely to open new, unexpected alleys for research. The letter is as relevant today as it was when published in 1972 and easily relates to citizen science projects.

The narrative reporting the outcomes from citizen science projects tends to use a fundamentally Apollonian view of science, which casts the researcher as an impartial observer and operates within a carefully designed methodology to guide the contributions from volunteer stakeholders. In the following sections we explore whether such a formal structure is always necessary for Citizen Science biodiversity projects to yield scientifically useful data. 


\section{Methodology}

As a case study, we use a real Citizen Science project, NatureSpot, selected because two of the authors, Cann and Nicholls, as project trustees identified benefits in credibility from supporting academic research. Before starting our exploration of this project we will briefly describe our methodological approach with a distinctly Apollonian style, the formality conveniently masks the post priori nature of this research and helps to develop a generalisation that may be applied when analysing other projects.

The initial contextual review of both project and data establishes an understanding of a priori knowledge and structure, that separates this work from a statistically driven data mining task which would uncover correlations without necessarily providing causation. In the most reduced form, our data comprises observations made of species (the observed) so there is a naturally encoded directionality which influences interpretation. This is why the title of this paper emphasises the observer, rather than the observed.

A directed data exploration used the reusable template methodology that has been developed by Palmer et al. (2019) as this allowed for iteration with revised and related data. The templates are written in r-markdown and 'knitted' with the data to produce analytical outputs. The allusions to knitting and weaving found in the markdown templates have their origins in the 'literate programming' concepts proposed by Knuth (1984) and relate to untangling narrative and programming threads to produce reproducible documents by combining the two threads into a coherent and readable analysis. The practical implementation using the RStudio IDE (RStudio Team, 2016) allows output to be created in PDF, HTML and Word formats with tables and figures cross referenced within the final document.

\section{A Citizen Science Context}

We explore the presumption of an Apollonian structure and a deficit in public knowledge when reporting outcomes from Citizen Science projects in Table 1 , in which we list the forms of Citizen Science Models tabulated by Shirk et al. (2012) and extend it by including both the Apollonian and Dionysian modes of public engagement. We are not trying to criticise either approach here, and acknowledge the value of a Apollonian mindset when organising scientific thinking, but as Szent-Györgyi (1972) pointed out in his letter, serendipitous advances arising out of Dionysian thinking are usually wrapped inside a post priori Apollonian structure for publication. 


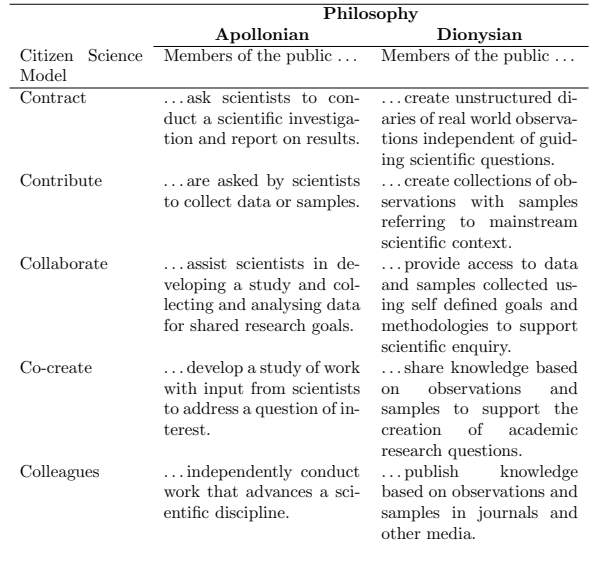

Table 1: Citizen Science Models compared against modes of public engagement. Extended from Shirk et al. (2012)

The Dionysian viewpoint has proved capable of making contributions to many domains of human activity, and Scheffer et al. (2015) called for more scientific training in this approach, but does it work with the citizen science paradigm? We explore this question by reviewing an established project, NatureSpot, that labels itself Citizen Science, and follows Dionysian principles where the contributors of natural history observations are self-motivated and self-appointed. Given the lack of formal structure it would be easy to write off the data collated as without scientific value, but we show that it is possible to infer a quasimethodology for subsets of data by deducing quantifiable observer behaviours and incorporating them into the interpretation.

There is already evidence for the value of such data, albeit using different terminology, in peer reviewed papers using longitudinal data collated by amateurs. Questions relating to post-industrial changes in the distribution and abundance of insects are hard to answer due to a paucity of data. However, a quantified decline in insects is documented by Vogel (2017) using the observations going back to 1905 made by the Krefeld Entomological Society in Germany, and other sources. The collections and observations used by Vogel were created a priori to the questions that they are now being used to answer, providing an elegant demonstration of the value that can arise from amateur enthusiasts.

\section{The NatureSpot Project}

NatureSpot is a registered charity, established in 2010, with the aim of increasing knowledge of the biodiversity of Leicestershire and Rutland, Watsonian Vice County VC55. Vice Counties have their origin in the work of botanist Watson (1873) and differ from the political county boundaries in that they remain constant through time facilitating comparative interpretation of biodiversity changes. Currently digital versions of the Vice Counties are available through the National Biodiversity Network (2018).

The NatureSpot website www.naturespot.org.uk is designed to collect VC55 bio- 
logical records submitted by the public. A profile has been created for the 6,700 species recorded with images along with a species account. Wildlife recording on NatureSpot is linked to the national iRecord system run by the Biological Records Centre at the Centre for Ecology and Hydrology. August et al. (2015) describe iRecord and other systems including how the data is securely stored and how it is passed to national recording schemes. The number of NatureSpot records has increased year on year, as has the number of recorders, and by November 2019, 249,322 records had been received from 1,214 recorders. Figure 1 shows Taxon groups with more than 3,000 records as a graphical depiction of their relative popularity.

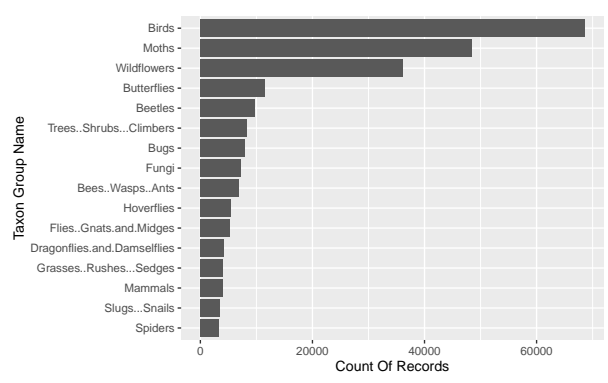

Figure 1: Taxon groups with more than 3,000 records. A graphical depiction of the numbers of records by taxon group which highlights their relative popularity.

High data quality is a key objective since, as in all projects with a citizen science projects element, confidence in the data is a prerequisite to the acceptance of any inferences made using it. There is considerable research activity exploring the issues of citizen science data quality, and research by Bates et al. (2013), Sullivan et al. (2009), Kosmala et al. (2016), Wiggins et al. (2011), Vantieghem et al. (2017), Isaac and Pocock (2015) all agree that the best projects can equal the quality of professional researchers. A positive linkage between data volume and the probability of academic publication was observed by Theobald et al. (2015) in their review of the potential for citizen science contributions in biodiversity research. Their work suggests that even 'messy' citizen science datasets are valuable if sample sizes are large, as variation among observers can be reconciled statistically.

In the following sections we explore the data statistically from two viewpoints; from that of the contributors; and then from the taxa observed.

\section{The Network of Contributors}

At a discrete level our data comprises observations that may be represented as an edge (a connection) between an observer node and a taxon node. We show below that this data representation uncovers relationships and groupings that may be analysed using a more formal Apollonian style approach. We thus start to bridge the gap between personally driven observations of nature and the need for structure in the analytic process. Individually, the NatureSpot contributors have no way to control the overall patterns that collectively emerge from the 
data, although we do know that external constraints confine observations to those made within the Leicestershire boundary, but no such constraints are made to the observers.

The work by Tumminello et al. (2011) describes how networks may be used to represent relationships in complex systems while preserving the underlying statistical validity. Thus network characteristics such as clusters and connections are manifestations of the real world presenting as features within the data. The NatureSpot data lends itself to a bipartite network visualisation of observers and species that may be represented as network nodes (vertexes) and observations as links (edges). We will use the terminology nodes and links here, but note that the alternate descriptions are frequently used in the supporting references. Bipartite refers to the presence of only two types of nodes, observers and taxon, where observations are represented by links. Note that, quite naturally the links are always between nodes of different types, a property of bipartite networks that will be used to good effect in the analysis that follows. Manipulation of large networks, while mathematically complex, is well-supported within the $\mathrm{R}$ analytical language (R Core Team, 2017), see Tyner et al. (2017), and we use it here to create powerful data driven visualisations, that help to build an understanding of the knowledge buried inside the data. In this narrative we focus on selected outputs from our analysis, but full listings of the analytical code and anonymised data are made available in the Open Science Foundation repository at https://osf.io/kfyw6/.

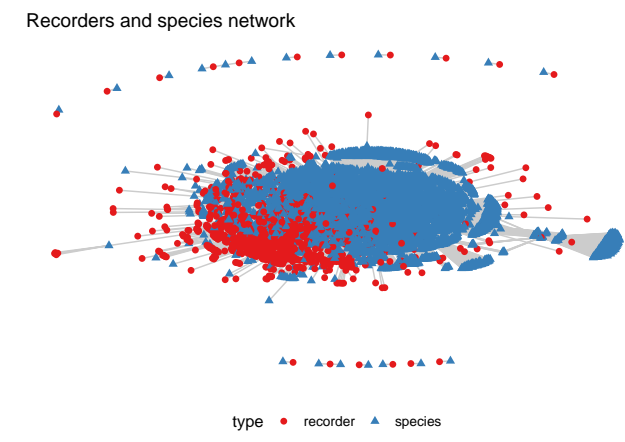

Figure 2: A network view of the records and recorders shows how they cluster into a single super group rather than multiple sub-clusters around recorder or taxon related factors.

Figure 2 comprises a network view of all available records. The nodes are coloured and shaped to separate species and recorders, and are placed according to a Fruchterman-Reingold algorithm (Fruchterman and Reingold, 1991). In this layout each node tries to repel every other node, and the links pull in opposition. While there is a random element detailed layout, repeated application of the rule will always form the same general patterns.

As we have an a priori understanding of the NatureSpot data, we expect recorders of many species to gravitate to the centre of clusters, while those who record only a few species to end up round the edge. A similar argument applies to the species, with the most recorded near the centre. Isolated groups within the 
network will tend to be repelled to the edges. Our interpretation of Figure 2 is that most taxon are seen by multiple recorders who record multiple taxon. The isolated nodes around the edge require investigation, but we make the assumption that these nodes should be eliminated from our analysis as they are rare species observed by recorders who have no track record of regular contributions.

Had recorders behaved in cliques split either by taxon groups or social groups we might have expected this to be reflected in the Fruchterman-Reingold layout revealing distinct clusters or communities in the disposition of the nodes and links. This can be explained conceptually as follows: starting from a random position, each node tries to repel every other node, while the edges may be thought of elastic bands that pull in opposition. The nodes and edges settle into positions where these opposing forces are in equilibrium. Thus groups of nodes with a greater number of links are held closer than nodes with fewer links. While there is a random element due to the starting position, repeated application of the rule will always form the same general patterns and clusters. While there are some visible groups of taxon due to the contributions of a few recorders, and a few isolated double and triple node networks, overall the observations link into a single super cluster, a feature we confirmed by using the deterministic walk-trap algorithm on the network as reported in the supporting documentation (Fortunato and Hric, 2016, E T H Zurich Department of Mathematics, 2015)

\section{Network Statistics}

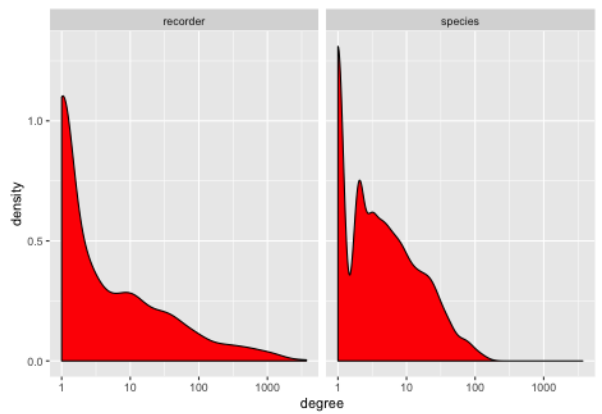

Figure 3: Degree: The number of connections as a proxy for expertise and abundance. Note the logarithmic scale of the x-axis.

Degree in network terminology, is the number of links to a particular node and in this case it represents the relationship between observer and the observed. In Figure 3 we can see from the frequency density plots of degree, that many recorders only see a few species and there are many species that have only been seen by a few recorders. Care is needed in the interpretation of this figure as we cannot assume that all recorders record in all taxon groups, and there are very different numbers of taxon within each taxon group. We already have a suggestion from Figure 2, that recorders do not form obvious clusters on taxon or recorder divisions, but even with these caveats, we suggest that degree may be used as a reasonable proxy for assigning categories equating to expertise and 
abundance within the data.

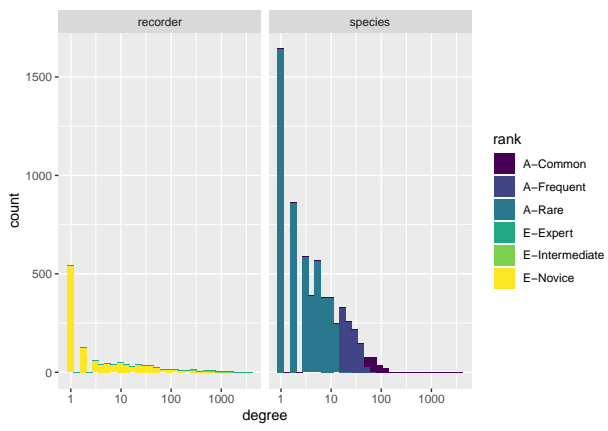

Figure 4: The k-means algorithm is used here to partition degree into three groups each for recorder and species: (A) abundance and (E) expertise. Experts see more species, and rare species are seen less frequently.

In Figure 4 the distribution of degree is represented by a histogram of the the results using the k-means algorithm (Maindonald, 2008). The k-means algorithm effectively decomposes a distribution into a predefined number of separate normal distributions. The algorithm has a random starting point, and delivers most consistent results when the normal distribution is an appropriate model for the underlying data, as in this case. Empirical trials found that splitting the distributions into three groups gave classifications that were reasonable sized subsets of the data.

These groups we named: Common, Frequent, Rare; and Expert, Intermediate, Novice. The choice of names for the split is suggestive of the interpretation; and the results fit with our opinion that expert recorders reliably observe more species than novices and are more cautious in their pronouncement of an identification. We therefore expect this to be reflected as a higher degree in the records with fewer rejected observations of rare species by expert record verifiers.

At the other end of the scale, we expect novices to observe fewer species and have a higher proportion of rejected observations of rare species. Intermediate recorders we presume to fall between these two groups, and we would like to think that there is a progression from novice to expert over time.

We interpret the shape of Figure 2 as being dominated by the contributions made by expert bird recorders along with their observations of other taxon groups. We justify this by applying counterfactual reasoning as proposed by Pearl (2019) to clarify causal relationships, and suggest that this case is simple enough to explore without resorting to supporting mathematical description. If the observers of non Bird taxon groups were dominated by recorders who largely ignored birds, then we would have expected clusters of such observers to have manifested themselves in the network view. No such differentiation of clusters are found, indicating that birds are recorded by most observers in addition to other taxon groups. Given these observations, we explore the taxon group of Birds in more detail. 


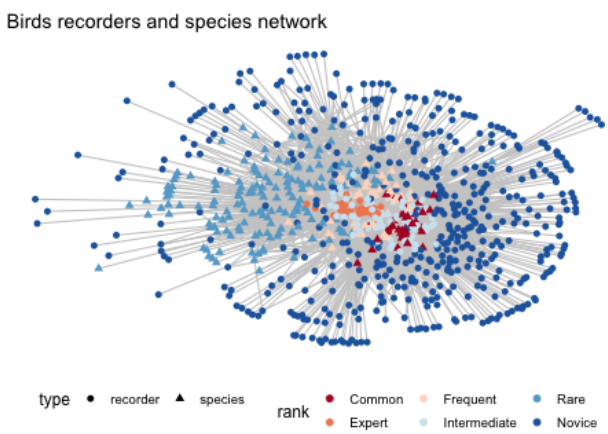

Figure 5: A network view of bird recording with k-means classifications shown. The layout shows that experts cluster toward the centre and are associated with the rarer species, suggesting these classifications are a reasonable proxy.

Figure 5 provides a more detailed picture of Bird records using the classifications just described. The node shapes indicate recorders and species. Colours are used to indicate the k-means derived clusters. It is evident that experts sit in the centre of the network, and all categories of species sit close to experts. Our intermediate recorders sit a little further out, novices cluster around the common species and form a more generalised halo characterised by few links. We can also confirm by examining the annotated data by hand, we agree with the identification of experts using this method. Given the simplicity of the analysis, we suggest degree may be a useful metric to consider using when analysing citizen science data, and so may be a tool to assign a reliability weighting to observers and their records.

\section{Wildlife As Seen Through NatureSpot}

There are clear preferences for recording particular taxon groups that need to be examined in any analysis of the data. Figure 1 revealed the extent of this bias within the data and shows how birds, moth and wild flowers numerically dominate the observations. An obvious question is: why this bias? And is this bias universal or is it peculiar to the UK Midlands? We cannot attribute cause, but we can search for other related factors such as geospatial distribution.

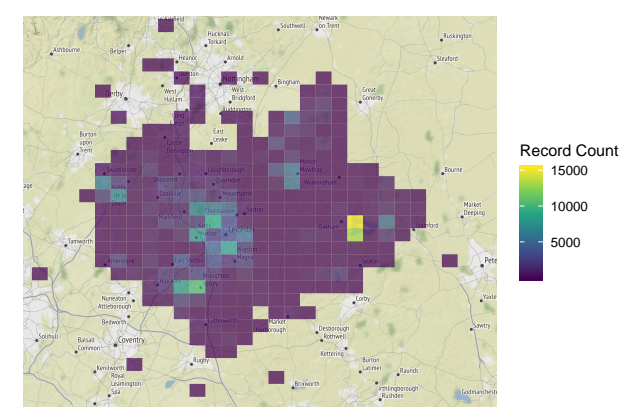

Figure 6: 2D histogram of the spatial distribution of popular group records. 
Figure 6 shows the overall distribution of popular group records in Figure 1 filtered from the source data. In Leicestershire \& Rutland, a distinctive pattern is perceived in the records for most taxon groups for reasons that we cannot fully explain. To the West of the county Bradgate County Park is well recorded, and there is also a peak in the East around Rutland Water Nature Reserve. Both areas are well visited and one expects the pattern of records. Not obvious, is why there is a well recorded region in the West central area with an adjacent sparsely recorded area to the East central area? No correlations with public spaces or land use were found to explain the pattern.

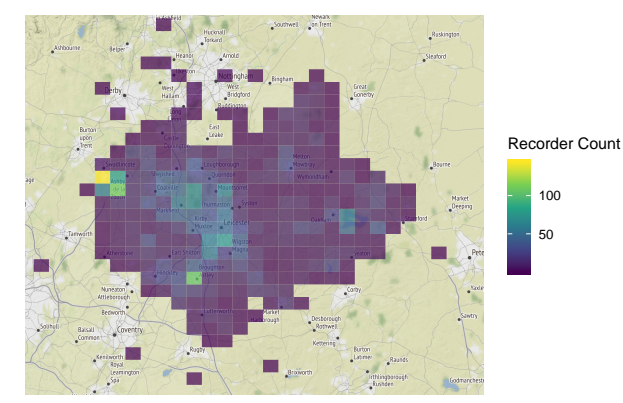

Figure 7: 2D histogram of the spatial distribution of popular group recorders.

The overall distribution of recorders helps to uncover a relationship between the recorded and recorder. Do we have a few recorders responsible for most of the records or many recorders more or less equally contributing to the overall pattern? Comparing Figure 6 with Figure 7 visually we note that Bradgate Park has more recorders than Rutland Water Nature Reserve, but otherwise the pattern remains broadly similar.

\section{Discussion}

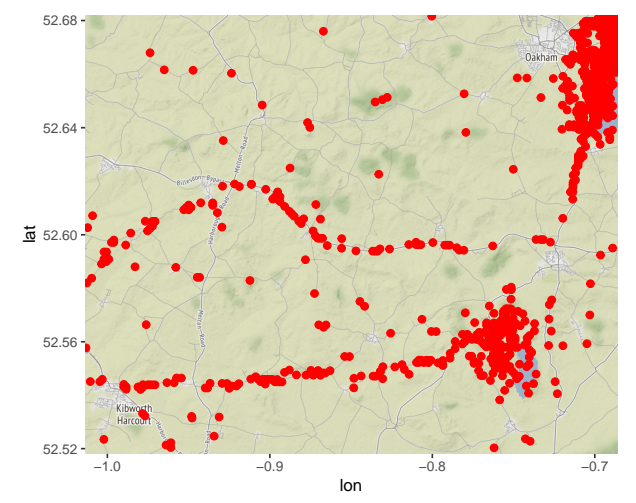

Figure 8: Distribution of Buzzard records showing the alignment with roads away from Rutland Water nature reserve and Eye Brook reservoir.

How can these observations be reconciled? A possible explanation lies in the idea that our Citizen Scientists have preferred locations that relate more closely 
to their regular haunts, unrelated to underlying biodiversity. If this is true then we might expect records of easily observed species to strongly correlate with particular locations.

A pattern that may be attributed to this hypothesis may be seen in Figure 8 which depicts observations of Buzzards. In the UK this large raptor species is easily identified with the naked eye at close range and at long range with binoculars as there are no plausible confusion species. There are the expected large clusters of observations around Rutland Water and Eye Brook Reservoir area which are frequented by birdwatchers. Away from these areas observations strongly align with roads, so observations are presumed to be made by car drivers. Figure 9 reveals a very similar distribution for the Red Kite another visually prominent species readily identified by its colour, hovering flight, and strongly forked tail.

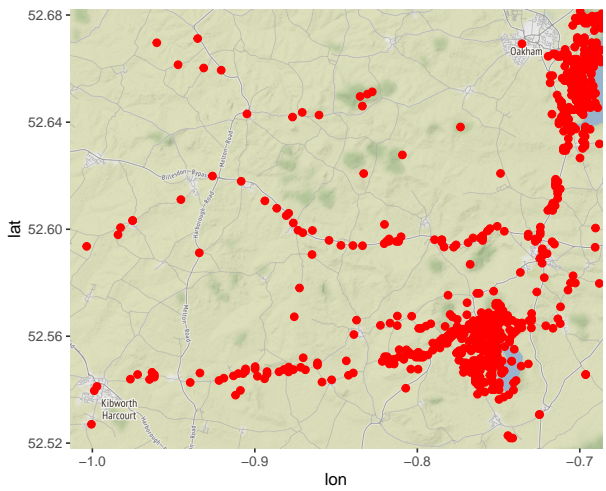

Figure 9: Distribution of Red Kite records showing the alignment with roads away from Rutland Water nature reserve and Eye Brook reservoir.

These observations draw us to the conclusion that most of the Citizen Scientists who contribute to NatureSpot are recorders of opportunity, rather than methodology, and that submissions are strongly driven by preferred locations and serendipitous observations. Understanding this behaviour is the first step towards producing models that account for the bias introduced by the lack of formal methodology. In the case of roadside observations, a quasi methodology arises where observations contained within long polygons that approximate to the roadside may constitute a representative sample for statistical analysis. Both these species are known to take carrion, so may use road kill as a food source, and sit on adjacent perches while digesting food. Thus busier roads may present a greater feeding opportunity, but more work is needed to explore this possibility.

A recent paper Falk et al. (2019), Evaluating the ability of citizen scientists to identify bumblebee (Bombus) species concludes that ...'The finding is strongly suggestive of a changing role of recording schemes, with a movement away from simply harvesting and collating data from known recorders, towards a more inclusive teaching and mentoring role.' Our data support this conclusion as we find the submission of records does not follow the expected distribution where novice recorders flood the system with records of common species. The question 
of how we encourage the progression from novice to expert remains open, but verification, feedback and mentoring must be a crucial role for citizen science biological recording schemes.

\section{Conclusions}

Returning to the initial question: 'Does Citizen Science biological recording tell us as much about the recorders as biodiversity?' The answer is yes.

The lack of a formal data collation methodology along with the existence of biases due to unknown causes are problematic when interpreting the underlying biodiversity. However, the large size of the data allows for statistical explorations that otherwise would not be practicable. The findings of this work show that proxies for observer expertise and geospatial species distribution can be derived from the data and allow insights into recorder behaviour that add value to the overall interpretation. Our findings suggest that good citizen science projects may be designed around Dionysian principles by appealing to individual stakeholder motivations, complementing the structured methodology preferred by the Apollonian approach.

It is clear that the data is not equivalent to that which is collected with a rigorous methodology, but this does not mean that it is without scientific value. Other observational branches of science such as geology, meteorology and astronomy have to collect data from sources over which they have no control so we suggest that we have to take a similar perspective with the data from NatureSpot and use statistical analysis to compensate for the behaviour of our observers. We also have the advantage that we can interact with our contributors and may have the long term advantage of being able to influence their behaviour.

The bipartite network analysis helped to clarify relationships between the observer and observed also providing an analytic method for deriving proxies of expertise and abundance. This work only touched upon the power of counterfactual reasoning when analysing observational data of this type when interpreting the clusters (or lack of them) in the network views. Future work will develop this approach further.

Beyond the data acquisition aspect of biological recording, the NatureSpot model of photo gallery based species identification accompanied by key identification points has proved to be immensely popular and must therefore be providing some of the intangible benefits of Cultural Ecosystems Services to participants. As a proxy measure, in 2019 the website received over 2.5 millionpage views from a wide geographical area according to Google Analytics. Even if visitors choose not to submit an in-area (Leicestershire and Rutland) biological record, by encouraging people to connect with nature through observing the natural world the NatureSpot citizen science project has considerable impact. 


\section{Acknowledgements}

The authors thank Sue Timms (Principal Ecologist) Leicestershire County Council, for advice during the preparation of this paper and EPSRC grant: EP/R513088/1 for funding the doctoral research on which this work is based.

\section{References}

August T, Harvey M, Lightfoot P, Kilbey D, Papadopoulos T, Jepson P. 2015. Emerging technologies for biological recording. Biological Journal of the Linnean Society 115: 731-749. ISSN 00244066.

Bates AJ, Sadler JP, Everett G, Grundy D, Lowe N, Davis G, Baker D, Bridge M, Clifton J, Freestone R, Gardner D, Gibson C, Hemming R, Howarth S, Orridge S, Shaw M, Tams T, Young H. 2013. Assessing the value of the Garden Moth Scheme citizen science dataset: how does light trap type affect catch? Entomologia Experimentalis et Applicata 146: 397. ISSN 00138703.

E T H Zurich Department of Mathematics. 2015. R: Hierarchical Clustering. URL https://stat.ethz.ch/R-manual/R-patched/library/stats/html/ hclust.html

Falk S, Foster G, Comont R, Conroy J, Bostock H, Salisbury A, Kilbey D, Bennett J, Smith B. 2019. Evaluating the ability of citizen scientists to identify bumblebee (Bombus) species. PLoS ONE 14. ISSN 19326203.

Fish R, Church A, Willis C, Winter M, Tratalos JA, Haines-Young R, Potschin M. 2016. Making space for cultural ecosystem services: Insights from a study of the UK nature improvement initiative. Ecosystem Services 21: 329-343. ISSN 22120416.

Fortunato S, Hric D. 2016. Community detection in networks: A user guide. Physics Reports 659: 1-44. ISSN 03701573.

Fruchterman TM, Reingold EM. 1991. Graph drawing by force-directed placement. Software: Practice and Experience 21: 1129-1164. ISSN 1097024X.

Isaac NJ, Pocock MJ. 2015. Bias and information in biological records. Biological Journal of the Linnean Society 115: 522-531. ISSN 10958312.

Knuth DE. 1984. Literate Programming. The Computer Journal 27: 97-111. ISSN 0010-4620.

Kosmala M, Wiggins A, Swanson A, Simmons B. 2016. Assessing data quality in citizen science. Frontiers in Ecology and the Environment 14: 551-560. ISSN 15409309.

Maindonald JH. 2008. Using R for Data Analysis and Graphics Introduction , Code and Commentary. Technical report, Australian National University.

Milcu AI, Hanspach J, Abson D, Fischer J. 2013. Cultural ecosystem services: A literature review and prospects for future research. Ecology and Society 18. ISSN 17083087. 
National Biodiversity Network. 2018. Mapping - Watsonian Vice Counties National Biodiversity Network.

URL https://nbn.org.uk/tools-and-resources/nbn-toolbox/ watsonian-vice-county-boundaries/

Palmer P, Henshaw M, Lock R. 2019. A Modular Task Orientated Approach for the Analysis of Large Datasets. OSF 10.31219/osf.io/ys2vw .

Pearl J. 2019. Sufficient causes: On oxygen, matches, and fires. Journal of Causal Inference 7: 8. ISSN 21933685.

Pyle RM. 1993. Thunder Tree: Lessons from an Urban Wildland. Boston, MA: Oregon State University Press, 2011 edition. ISBN 978-0870716027.

R Core Team. 2017. R: A language and environment for statistical computing. R Foundation for Statistical Computing .

RStudio Team. 2016. RStudio. URL http://www.rstudio.com/

Sarukhán J, Whyte A. 2005. Millenium Ecosystem Assessment. Technical report, World Resources Institute, Washington, DC.

Scheffer M, Bascompte J, Bjordam TK, Carpenter SR, Clarke LB, Folke C, Marquet P, Mazzeo N, Meerhoff M, Sala O, Westley FR. 2015. Dual thinking for scientists. Ecology and Society 20. ISSN 17083087.

Shirk JL, Ballard HL, Wilderman CC, Phillips T, Wiggins A, Jordan R, McCallie E, Minarchek M, Lewenstein BV, Krasny ME, Bonney R. 2012. Public participation in scientific research: A framework for deliberate design. Ecology and Society 17: 20. ISSN 17083087.

Soga M, Gaston KJ. 2016. Extinction of experience: The loss of human-nature interactions.

Sullivan BL, Wood CL, Iliff MJ, Bonney RE, Fink D, Kelling S. 2009. eBird: A citizen-based bird observation network in the biological sciences. Biological Conservation 142: 2282-2292. ISSN 00063207.

Szent-Györgyi A. 1972. Dionysians and apollonians. Science 176: 966. ISSN 00368075 .

Theobald EJ, Ettinger AK, Burgess HK, DeBey LB, Schmidt NR, Froehlich HE, Wagner C, HilleRisLambers J, Tewksbury J, Harsch MA, Parrish JK. 2015. Global change and local solutions: Tapping the unrealized potential of citizen science for biodiversity research. Biological Conservation 181: 236-244. ISSN 00063207.

Tumminello M, Miccichè S, Lillo F, Piilo J, Mantegna RN. 2011. Statistically validated networks in bipartite complex systems. PLoS ONE 6: e17994. ISSN 19326203.

Tyner S, Briatte F, Hofmann H. 2017. Network Visualization with ggplot2. The $R$ Journal 9: 27-59. ISSN 2073-4859. 
Vantieghem P, Maes D, Kaiser A, Merckx T. 2017. Quality of citizen science data and its consequences for the conservation of skipper butterflies (Hesperiidae) in Flanders (northern Belgium). Journal of Insect Conservation 21: 451-463. ISSN 15729753.

Vogel G. 2017. Where have all the insects gone? Science 356: 576-579.

Watson HC. 1873. Topographical botany; being local and personal records towards shewing the distribution of British plants traced through the 112 counties and vice-counties of England and Scotland. [London]: Thames Ditton.

Wiggins A, Newman G, Stevenson RD, Crowston K. 2011. Mechanisms for data quality and validation in citizen science. In Proceedings - 7th IEEE International Conference on e-Science Workshops, eScienceW 2011. ISBN 9780769545981, 14-19. 